\title{
An extended generalized effective stress for active clays
}

\author{
Angelica Tuttolomondo ${ }^{1, *}$, Alessio Ferrari ${ }^{1}$, and Lyesse Laloui ${ }^{1}$ \\ ${ }^{1}$ Swiss Federal Institute of Technology in Lausanne, EPFL, Laboratory of Soil Mechanics, LMS, Station 18, CH 1015 Lausanne, \\ Switzerland
}

\begin{abstract}
An effective stress concept for active clays is formulated to improve the modelling of these materials under a variety of environmental conditions. In particular, the mechanical effects induced by a change in the chemical potential of pore water associated with a change of its pressure (even in the negative range), as well as a change of its chemical composition, are considered. Some implications of the proposed framework are shown with reference to shear strength results related to unsaturated conditions. The proposed approach allows to describe the shear strength envelope consistently by using a unique set of shear strength parameters irrespective of the pore water chemical composition and of the saturation state.
\end{abstract}

\section{Introduction}

Active clays are often one of the geological barriers within nuclear waste storage repositories or one of the layers within geosynthetic liners used in a variety of containment applications. As a result, in addition to being subjected to variations of the mechanical loads, they may be subjected to drying and wetting processes through the liquid or the vapor phase. Consequently, the pressure of the pore water can vary significantly (assuming also negative values) and variations in the chemical composition of the pore water can also take place.

The chemical potential of the pore water within the representative elementary volume of a geomaterial depends on its: (i) pressure (ii) temperature (iii) activity; activity, in turn, can be a function of pressure, temperature, chemical composition and of the presence of liquid-gas interfaces related to a pore water pressure lower than the pressure of the gas phase. Any variation in the chemical potential of pore water, related to one of the above-mentioned factors, typically affects the mechanical response of the involved geomaterial, with reference to shear strength and volumetric behaviour both (effects of pressure also in the negative range: e.g. [1], [2]; temperature's effects: e.g. [3], [4]; chemical composition's effects: [5], [6]). With specific reference to the chemical composition of the pore water, experimental studies available in literature show that the effect of its variation is the more significant the more active the clay is (see for example [7]).

An advanced geomechanical approach should take all these aspects into account for a comprehensive description of the mechanical behaviour of clays, especially if active. To this end, there is a need for: (i) an effective stress variable (defined from now on as the single mechanical stress variable) suitable for governing the strains of the solid skeleton, including the strains induced by a change of the chemical potential of the pore water; (ii) the definition of the stress variables (hydraulic or electrochemical in nature) and the corresponding stress-strain type constitutive relations necessary for the determination of the effective stress at any state; (iii) a suitable constitutive context [8].

This paper proposes to extend the concept of generalized effective stress [9] to active clays. In particular, the chemical composition of the pore water is determined by including also some of the ions necessary for the electroneutrality of the clay's particles. The pressure of the pore water is redefined as a function of the measurable or controllable matric suction. Finally, the formulation of the effective stress is updated and made dependent, among other factors, also on the chemical composition of the pore water. Compared to the existing formulation, the proposed concept of effective stress allows for define a suitable failure envelope unique for all saturation levels and all the pore water chemical compositions. In this paper, the performances of the framework are revealed by referring to shear strength data at negative pore water pressures (positive matric suction values).

\section{The extended generalized effective stress concept}

The conversion of a multiphase porous medium into an equivalent continuous medium can be achieved conveniently using the concept of generalized effective stress [9]. Within this framework, a possible definition of the mechanical stress variable is provided by the following expression:

$$
\sigma_{i j}^{\prime}=\sigma_{n e t, i j}+S_{r} s_{m} \delta_{i j}
$$

Corresponding author: angelica.tuttolomondo@epfl.ch 
where $\sigma_{n e t, i j}=\sigma_{i j}-u_{g} \delta_{i j}$ is the net stress tensor, $s_{m} \delta_{i j}=\left(u_{g}-u_{w}\right) \delta_{i j}$ is the matric suction stress tensor and $S_{r}$ is the degree of saturation.

Active clays are characterized by the presence of clay particles (for instance, montmorillonite) in which the chemical bonds between the different basic units are very weak. The structure of these particles can significantly vary depending on the environmental conditions. The electroneutrality is guaranteed also by the presence of cations on the external surfaces of the particles. These cations are named in this study nonmovable ions. In the presence of water molecules, the non-movable ions are dissociated from the particles and are located at distances from them that can reach up to $50 \mathrm{~nm}$ [10] (compatible with other factors including the space available and, eventually, the chemical composition of the bulk water). The properties of water are expected to remain constant, and typical of a liquid water, from the first nanometer onwards (e.g. $[11,12,13])$.

In the context of this study, the bulk water is the water which does not include non-movable ions because it is located at a sufficient distance from the surface of the particles. The pore water is the water having the typical properties of liquid water. The presence of a fraction of non-movable cations in the pore water affects its chemical composition, as well as the pore water pressure compared to the externally measured or imposed value.

By applying the principles of thermodynamics and geochemistry, as well as the concept of solute suction, to the representative volume of an active clay, it is possible:

a. To express the pressure of the pore water as follows:

$$
u_{p w}=u_{g}-s_{m}+s_{s, e}
$$

where $u_{g}$ is the gaseous pressure, $s_{m}$ is the externally measured (or imposed) matric suction and $s_{s, e}$ is an interior stress variable, named effective solute suction, related to the interaction of the clay particles with the water molecules. The effective solute suction curve is found to be affected by the chemical composition of the pore water. An increase of the salt concentration within the bulk water results in a decrease of the effective solute suction.

b. To rewrite the equivalent pore pressure in the following form

$$
u^{*}=\left(1-S_{r}\right) u_{g}+S_{r} u_{p w}
$$

and, in the light of equation (2), to define the extended generalized effective stress as

$$
\sigma_{i j}^{\prime}=\sigma_{n e t, i j}+S_{r}\left(s_{m}-s_{s, e}\right) \delta_{i j}
$$

where $s_{s, e} \delta_{i j}=\left(s_{s, p w}-s_{s}\right) \delta_{i j}$ is the effective solute suction stress tensor. At a given matric suction and saturation state, an increase of the salt concentration in the bulk water results in a decrease of the effective solute suction which in turn leads to an increase of the effective stress. This feature of the proposed effective stress model is in agreement with the experimental results showing an increase in strength as well as a decrease in volume when the concentration of salt in the pore water becomes higher [e.g. 5].

c. To analytically determine the value of the effective solute suction depending on the mineralogy of the geomaterial, the chemical characteristics of the water within the measuring or pressure control system (this water typically coincides with the bulk water), the degree of saturation and the state of compaction. The variation of the effective solute suction with respect to the degree of saturation is named effective solute suction curve. An example of effective solute suction curve obtained analytically by solving electroneutrality and thermodynamic equilibrium equations is provided in Fig. 1. The curve refers to a bentonite material characterized by a cation exchange capacity equal to $74 \mathrm{cmol} / \mathrm{kg}$ and a fraction of non-movable cations within the pore water equal to 0.15 (other assumed quantities: void ratio equal to 1 , solid unit weight equal to $2.67 \mathrm{Mg} / \mathrm{m} 3$ and $0.2 \mathrm{M}$ $\mathrm{NaCl}$ bulk water). The analytic results allow to propose a non-linear model for the description of the effective solute suction curve when the matric suction changes. The proposed model is described by the equation:

$$
s_{s, e}=a S_{r}^{b}
$$

where $a$ and $b$ can be considered, as a first approximation, material parameters. The effective solute suction curve model for the considered bentonite is provided in Fig. 1 ( $a=369 \mathrm{kPa}$ and $\mathrm{b}=-1.27)$.

The proposed framework makes the choice of the water retention and of the effective solute models, as well as the determination of the corresponding parameters, of fundamental importance.

In the following, the analysis of some experimental results according to the proposed framework is provided. The results highlight the advantages of the proposed approach as justification for its adoption. 


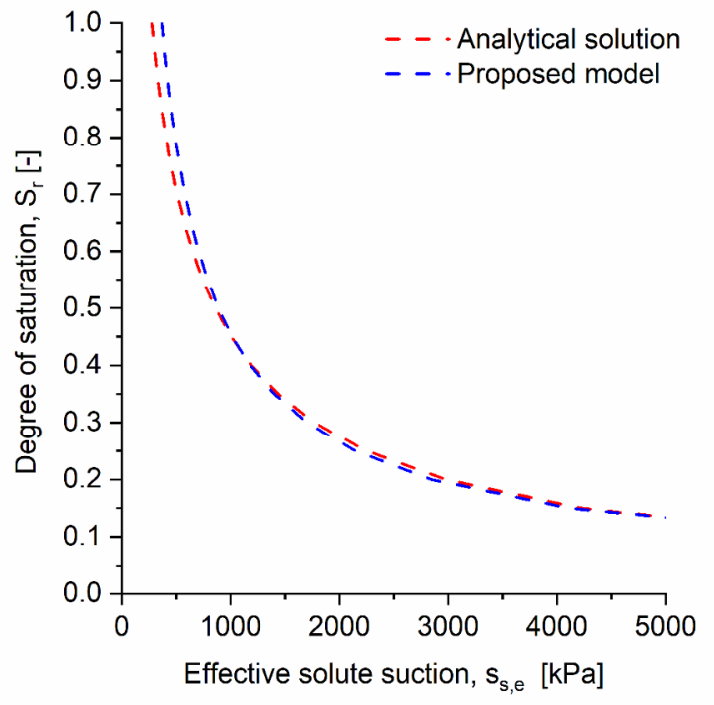

Fig. 1. Analytical solution and model of the effective solute suction curve for a bentonite.

\section{Implications of the adoption of the extended generalized effective stress for unsaturated states}

Replacing the definition of the extended generalized effective stress (equation (4)) in the Mohr-Coulomb criterion allows to express the shear strength $\left(\tau_{f}\right)$ of an active clay as

$$
\tau_{f}=c^{\prime}+\left[\sigma_{n e t, n}+S_{r}\left(s_{m}-s_{s, e}\right)\right] \tan \varphi^{\prime}
$$

where $\sigma_{\text {net }, n}$ is the net normal stress acting on the plane (i.e. the difference between the normal total stress and the gaseous pressure); $c^{\prime}$ and $\varphi^{\prime}$ are the effective shear strength parameters.

Fig. 2a and Fig. 2b plot the laboratory results for the determination of a retention curve (WRC) and of the shear strength failure envelope of Glacial Till (experimental data from [14]), both referred to a net stress of $25 \mathrm{kPa}$. The experimental data of the retention curve are interpreted according to the Nuth and Laloui's water retention model [15]. The elasto-plastic water retention model is characterized by five different parameters whose values are provided in Table $1\left(\mathrm{~s}_{\mathrm{e}}\right.$ is the air entry value, $S_{\text {res }}$ is the residual degree of saturation, $\mathrm{K}_{\mathrm{H}}$ is an elastic modulus for the definition of the elastic increment of the degree of saturation, $\beta_{\mathrm{H}}$ is the coefficient of compressibility for the plastic part of the degree of saturation and $s_{D 0}$ is the reference yield suction).

Experimental shear strength data are interpreted according to the generalized effective stress concept [9] and the extended generalized approach proposed in this study (Fig. 2b).

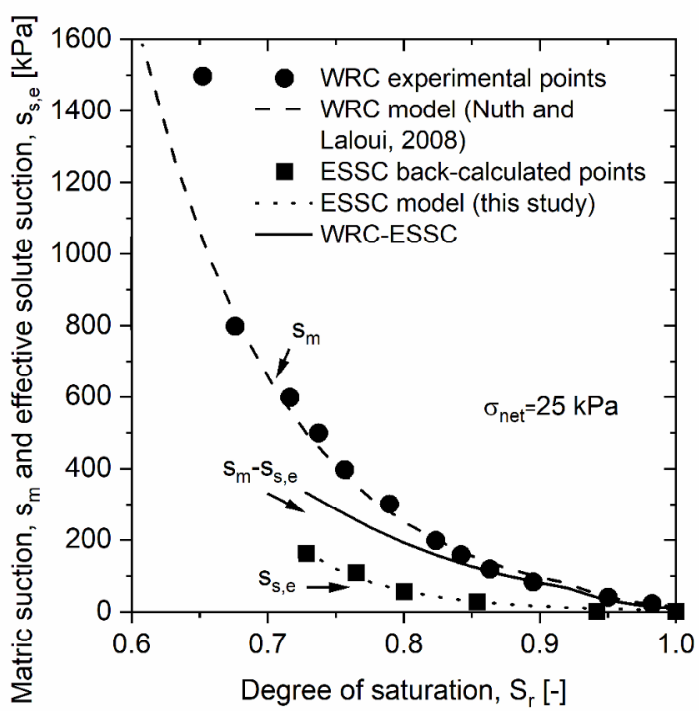

(a)

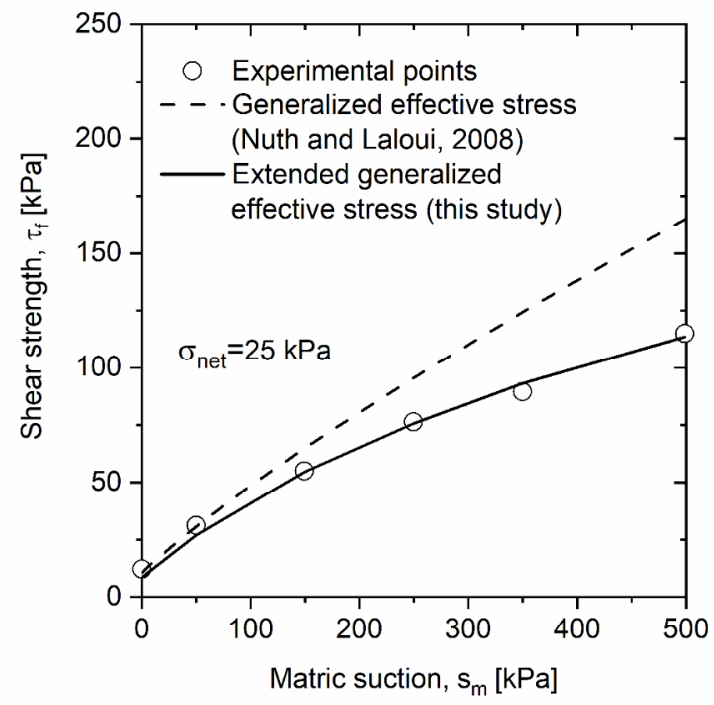

(b)

Fig. 2. Water retention curve, effective solute suction curve and shear strength envelope of Glacial Till tested at $\sigma_{\text {net }}=25$ $\mathrm{kPa}$. Experimental points from [14].

The extended generalized approach is adopted by calculating backwards the values of effective solute suction relative to each experimental point of the failure envelope. In particular, it is imposed that the experimental shear strength value coincides with the shear strength value provided by the Mohr-Coulomb criterion written as a function of the extended generalized effective stress. For this purpose, the saturated shear strength parameters (Table 1), the experimental measurements of matric suction at different failure conditions and the corresponding degrees of saturation obtained from the retention curves are used. 
Fig. 2a shows the back-calculated values of effective solute suction (negative values are set equal to zero) at different degrees of saturation (effective solute suction curve, ESSC). The results show to be well interpreted by the model proposed on the basis of the analytical results (parameters of the model are given in Table 1). The shear strength envelope according to the extended generalized approach (Fig. 2b) adopts as effective solute suction values those provided by the effective solute suction curve model. The increase in matric suction, and the corresponding reduction in the degree of saturation, results in a change in the chemical composition of the pore water. In fact, although the water content within the geomaterial is expected to decrease without a significant change in the chemical composition of the bulk water, the presence of the non-movable ions belonging to the pore water affects the chemical composition of it. Therefore, as the degree of saturation decreases, the effective solute suction increases following the expected theoretical trend.

Table 1. Parameters adopted in this study.

\begin{tabular}{|c|c|}
\hline \multicolumn{2}{|c|}{ Water retention curve } \\
\hline $\mathrm{s}_{\mathrm{e}}[\mathrm{kPa}]$ & 14 \\
\hline $\mathrm{S}_{\mathrm{res}}[-]$ & 0.15 \\
\hline $\mathrm{K}_{\mathrm{H}}[\mathrm{kPa}]$ & $2.2 \mathrm{E}+07$ \\
\hline$\beta_{\mathrm{H}}[-]$ & -17 \\
\hline $\mathrm{s}_{\mathrm{D} 0}[\mathrm{kPa}]$ & 78 \\
\hline \multicolumn{2}{|c|}{ Effective solute suction curve } \\
\hline $\mathrm{a}[\mathrm{kPa}]$ & 4.87 \\
\hline$b[-]$ & -11.14 \\
\hline \multicolumn{2}{|c|}{$\begin{array}{c}\text { Saturated shear strength } \\
\text { parameters }\end{array}$} \\
\hline$\varphi^{\prime}\left[{ }^{\circ}\right]$ & 23 \\
\hline$c^{\prime}[\mathrm{kPa}]$ & 0 \\
\hline
\end{tabular}

\section{Conclusions}

An extension of the generalized effective stress framework to active clays is proposed. This approach has the advantages of the generalized effective stress concept reported in [9]; among which, it allows a direct transition between saturated and unsaturated states by using a single set of shear strength parameters; it enables to implicitly accounting for the increase in strength associated with an increase in matric suction. The nonlinearity of the shear strength envelope in the shear strength-matric suction plane is associated with a variation of the matric suction (and corresponding variation in the degree of saturation, in accordance with the retention curve) and with a variation of the chemical composition of the pore water.

The proposed approach, among its inbuilt features, allows to consider the mechanical effects of a change in chemical composition of its water even if it is due, at a given degree of saturation, to a change in salt content due to diffusion.

\section{References}

1. A. Ferrari, V. Favero, P. Marschall, L. Laloui. International Journal of Rock Mechanics and Mining Sciences. 72, (2014)

2. V. Escario, J.F.T. Jucá. Proceedings of the 12th International Conference on Soil Mechanics and Foundation Engineering, Rio de Janeiro. 2, (1989)

3. C. Cekerevac, L. Laloui. International Journal for Numerical and Analytical Methods in Geomechanics. 28, (3) (2004)

4. L. Laloui, S. Salager, M. Rizzi. Acta Geotechnica. 8, (5) (2013)

5. C. Di Maio. Géotechnique. 46, (4) (1996)

6. N. Mokni, E. Romero, S. Olivella. Géotechnique. 64, (9) (2014)

7. C. Di Maio, G.B. Fenelli. Géotechnique. 44, (4) (1994)

8. L. Laloui, M. Nuth. Computers and Geotechnics. 36, (1-2) (2009)

9. M. Nuth, L. Laloui. International Journal for Numerical and Analytical Methods in Geomechanics. 32, (7) (2008)

10. K. Terzaghi, R. Peck, G. Mesri. Soil Mechanics in Engineering Practice. Wiley, 1996.

11. L. Cheng, P. Fenter, K.L. Nagy, M.L. Schlegel, N.C. Sturchio. Phys Rev Lett. 87, (15) (2001)

12. S. Adapa, A. Malani. Sci Rep. 8, (1) (2018)

13. C. Tournassat, Y. Chapron, P. Leroy, M. Bizi, F. Boulahya. J Colloid Interface Sci. 339, (2) (2009)

14. S.K. Vanapalli, D.G. Fredlund, D.E. Pufahl, A.W. Clifton. Canadian Geotechnical Journal. 33, (1996)

15. M. Nuth, L. Laloui. Computers and Geotechnics. 35, (6) (2008) 